\title{
A GENERALIZED INVERSIVE ALGORITHM
}

\author{
BY D. H. LEHMER
}

In a previous note* we considered an algorithm for constructing the $n$th member of any infinite class $C$ of positive integers, an algorithm used by Brun $\dagger$ for the non-tentative calculation of the $n$th prime. In this note we give a generalization in two directions of the Brun algorithm. In the first place it is made applicable to any denumerable set of elements and secondly a parameter $\left(m_{0}\right)$ is introduced which allows the algorithm to start from a more advantageous position than in the case of Brun, where one starts by saying that the $n$th prime is not less than $n$.

The algorithm may be presented in the following form:

Let $S: e_{1}, e_{2}, e_{3}, \cdots$, be a class of ordered elements.

Let $C: e_{a_{1}}, e_{a_{2}}, e_{a_{3}}, \cdots,\left(a_{i}<a_{i-1}\right)$, be any infinite subclass of $S$. For $x$ an integer $\ddagger>0$, we define $\theta(x)$ as the number of elements of $C$ belonging to the set of the first $x$ elements of $S$. For $x \leqq 0$, we define $\theta(x)=0$. Let $m_{0}$ and $n$ be any positive integers. We then form the sequence

$$
m_{1}, m_{2}, m_{3}, \cdots,
$$

defined by

$$
\begin{aligned}
& m_{1}=n-\theta\left(m_{0}\right), \\
& m_{2}=n-\theta\left(m_{0}+m_{1}\right), \\
& m_{3}=n-\theta\left(m_{0}+m_{1}+m_{2}\right), \\
& \cdot . \cdot . \cdot . \cdot . \cdot . \\
& m_{r}=n-\theta\left(s_{r}\right),
\end{aligned}
$$

where we have written $s_{r}$ for $m_{0}+m_{1}+\cdots+m_{r-1}$. We may then state the following theorem.

Theorem. The terms of (1) do not differ in sign, nor do they increase in absolute value. There exists moreover an integer $k$ such

* This Bulletin, vol. 38 (1932), pp. 693-694.

† Kongelige Norske Videnskaps-selskabet, vol. 4, pp. 66-69.

$\ddagger$ If $x$ is not an integer one could define $\theta(x)=\theta([x])$. 
that $m_{\nu} \neq 0$ for $\nu<k$, while $m_{\nu}=0$ for $\nu \geqq k$. If $m_{1}>0$, then $e_{s_{k}}$ is the nth member of the class $C$. If $m_{1}<0$, then $e_{s_{k-1}}$ is the $\left(n-m_{k-1}\right)$ st member of $C$. If $m_{1}=0$, the nth member of $C$ is $e_{h}$, where $n \leqq h \leqq m_{0}$.

Proof. If $m_{1}=0$, then equations (2) are all identical and reduce to

$$
n=\theta\left(m_{0}\right),
$$

all the other $m$ 's are zero. The theorem then follows at once with $k=1$, the last statement following from (3) which tells us that $n$ members of $C$ have subscripts $\leqq m_{0}$.

From the definition of $\theta(x)$, we have for $x>y$,

$$
0 \leqq \theta(x)-\theta(y) \leqq x-y .
$$

For $m_{1} \neq 0$ we consider the difference

$$
m_{r-1}-m_{r}=\theta\left(s_{r}\right)-\theta\left(s_{r-1}\right)=\theta\left(s_{r-1}+m_{r-1}\right)-\theta\left(s_{r-1}\right) .
$$

Using (4) we see that if $r \geqq 2$,

$$
\begin{aligned}
& m_{r-1}=0 \text { implies } m_{r}=0, \\
& m_{r-1}>0 \text { implies } 0 \leqq m_{r-1}-m_{r} \leqq m_{r-1}, \\
& m_{r-1}<0 \text { implies } 0 \geqq m_{r-1}-m_{r} \geqq m_{r-1} .
\end{aligned}
$$

From these equations we see that the $m$ 's do not differ in sign. Also (7) shows that the $m$ 's do not increase if positive, while (8) shows that they do not decrease if negative. Therefore the sequence $\left|m_{r}\right|,(r=1,2,3,4, \cdots)$, is nonincreasing. If possible suppose that $\left|m_{r}\right|>0$, for all $r>0$; then $s_{r}=m_{0}+m_{1}+m_{2}+\cdots+m_{r-1}$ would either increase or decrease indefinitely according as $m_{1}>0$ or $<0$. In the first case, since $C$ is an infinite class, $\theta\left(s_{r}\right)$ would also increase without limit, so that for $r$ sufficiently large $m_{r}=n-\theta\left(s_{r}\right)$ would be negative, contrary to fact. In the second case $s_{r}$ would ultimately become negative so that $\theta\left(s_{r}\right)=0$. For such an $r$ we would have $m_{r}=n$. But $n$ is positive, while $m_{r}$ is not. Hence $\left|m_{r}\right|$ becomes and remains zero for all sufficiently large $r$, say $r \geqq k$.

To complete the proof of the theorem set $r=k$ in (5) and obtain

$$
m_{k-1}=\theta\left(s_{k-1}+m_{k-1}\right)-\theta\left(s_{k-1}\right) .
$$


If $m_{1}$ and hence $m_{k-1}$ is positive, (9) tells us that all the $m_{k-1}$ members of $S: e_{s_{k-1}+1}, \cdots, e_{s_{k-1}+m_{k-1}}$ belong to $C$. In particular the last one which is $e_{s_{k}}$ belongs to $C$. Moreover $m_{k}=n-\theta\left(s_{k}\right)=0$. Hence $e_{s_{k}}$ is the $n$th member of $C$. If $m_{1}$ and hence $m_{k-1}$ is negative, (9) tells us that all the $m_{k-1}$ members of $S: e_{s_{k+1}}, \cdots, e_{s_{k-1}}$ belong to $C$. In particular $e_{s_{k-1}}$ is a member of $C$. But $m_{k-1}=n-\theta\left(s_{k-1}\right)$ or $\theta\left(s_{k-1}\right)=n-m_{k-1}$; hence $e_{s_{k-1}}$ is the $\left(n-m_{k-1}\right)$ st member of $C$. Thus the proof is complete.

If $S$ and $C$ are wholly arbitrary one may show by means of examples that the conclusion of the theorem is the best that can be obtained from the hypothesis. Additions to the hypothesis make the last two statements of the theorem more precise. For example, if we assume that no two consecutive members of $C$ are consecutive members of $S$, it is easy to prove that $e_{s_{k-1}}$ is the $(n-1)$ st member of $C$ in case $m_{1}<0$.

LEHIGH UNIVERSITY

\title{
A NEW CLASS OF FUNCTIONS OF TWO VARIABLES INVOLVING BESSEL FUNCTIONS OF HALF AN ODD INTEGER*
}

\author{
BY N. A. HALL
}

The evaluation of certain integrals arising in the theory of the conduction of heat between two media of different conductivities $\dagger$ suggested the consideration of the expansions:

$$
\begin{aligned}
\sin \left[\alpha\left(1+x^{2}\right)^{1 / 2}-\beta x\right] & =\sum_{n=0}^{\infty} x^{n} S_{n}(\alpha, \beta), \\
\cos \left[\alpha\left(1+x^{2}\right)^{1 / 2}-\beta x\right] & =\sum_{n=0}^{\infty} x^{n} C_{n}(\alpha, \beta), \\
\exp i\left[\alpha\left(1+x^{2}\right)^{1 / 2}-\beta x\right] & =\sum_{n=0}^{\infty} x^{n} E_{n}^{(1)}(\alpha, \beta), \\
\exp -i\left[\alpha\left(1+x^{2}\right)^{1 / 2}-\beta x\right] & =\sum_{n=0}^{\infty} x^{n} E_{n}^{(2)}(\alpha, \beta) .
\end{aligned}
$$

* Presented to the Society, November 30, 1935.

† G. Green, Philosophical Magazine, (7), vol. 18 (1934), p. 631. 